A $\mathrm{Cublications} \underset{G}{G}$ Rec. Nat. Prod. 14:2(2020) 160-165

\title{
Chemical Composition, Antibacterial, Antioxidant and Cytotoxic Activities of the Essential Oil of Dianella ensifolia
}

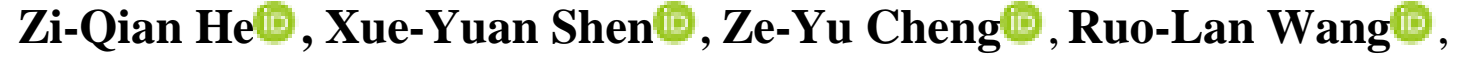
Peng-Xiang Lai and Xiang Xing

\author{
Marine College, Shandong University, Weihai 264209, China \\ (Received July 08, 2019; Revised September 23, 2019; Accepted September 24, 2019)
}

\begin{abstract}
The essential oil (EO) was isolated from aerial parts of Dianella ensifolia (L.) DC by hydro-distillation and its chemical constituents was determined by GC-FID and GC-MS. In total, sixty-three compounds comprising $97.2 \%$ of the EO were identified. The major compounds in D. ensifolia $\mathrm{EO}$ were found to be allo-aromadendrene (7.3\%), geranylacetone (6.2\%), hexahydrofarnesyl acetone (4.4\%), longifolene $(4.2 \%)$ and $\beta$-caryophyllene (4.0\%). Besides, the essential oil was evaluated for its antibacterial activity by disc diffusion and broth microdilution method. The D. ensifolia $\mathrm{EO}$ exhibited a potential broad-spectrum in vitro antibacterial activity against both Gram-positive and Gram-negative bacteria. Also, antioxidant activities of the EO were examined by employing DPPH, ABTS as well as FRAP assays. A weak to moderate antioxidant activity of the EO was observed. Furthermore, in vitro cytotoxic activity evaluation against the cell lines HepG2 and MCF-7 by MTT method showed a potent cytotoxicity with $\mathrm{IC}_{50}$ values of $61.35 \mu \mathrm{g} / \mathrm{mL}$ and $56.53 \mu \mathrm{g} / \mathrm{mL}$, respectively.
\end{abstract}

Keywords: Dianella ensifolia (L.) DC; essential oil; antibacterial activity; antioxidant activity; cytotoxic activity. () 2019 ACG Publications. All rights reserved. (C) 2019 ACG Publications. All rights reserved.

\section{Plant Source}

The fresh aerial parts of Dianella ensifolia (L.) DC were collected in May 2018 from Guigang in Guangxi Province of China. The plant material (voucher specimen NO.0180036) was identified by Prof. Hong Zhao and deposited at the Laboratory of Botany of Marine College, Shandong University, China.

\section{Previous Studies}

Dianella ensifolia (L.) DC., an evergreen perennial herb belonging to the genus Dianella (Liliaceae family), is widely distributed in the south of China. In Chinese traditional medicine, $D$. ensifolia is widely used for treating lymphangitis, tinea and carbuncle sore abscess [1]. Previous phytochemical investigations conducted with various parts of Dianella ensifolia have resulted in the

\footnotetext{
* Corresponding author: E-Mail: sdeduxx@163.com, Phone +86-631-5688303.
} 
isolation and identification of triterpenoids [2] and flavans [3] from the roots, dihydronaphtaquinone and quinones from the leaves [4] and propanes from the methanol extract of the plant $[5,6]$.

\section{Present Study}

To the best of our knowledge, no study regarding $D$. ensifolia essential oil has been performed. Here we analyzed the chemical constituents of the essential oil extracted from the aerial parts of $D$. ensifolia and evaluated its antibacterial, antioxidant and cytotoxic activities (the experiments details were provided in Supporting Information). The air-dried aerial parts of $D$. ensifolia were isolated by hydrodistillation method and the EO was obtained in the yield of $0.28 \%$ (v/w). Sixty-three compounds accounting for $97.2 \%$ of the total essential oil were determined by GC-FID and GC-MS (Table 1). The predominant components of $D$. ensifolia EO were identified as allo-aromadendrene (7.3\%), geranylacetone (6.2\%), hexahydrofarnesyl acetone (4.4\%), longifolene (4.2\%) and $\beta$-caryophyllene $(4.0 \%)$. It is interesting to note that these predominant compounds had been demonstrated to exhibit extensive biological activities. Allo-aromadendrene, the epimer of the tricyclic aromadendrene, has been demonstrated to significantly inhibit cell growth and proliferation in the highly malignant $+\mathrm{SA}$ mammary epithelial cells at a dose of $20 \mu \mathrm{M}$ [7], and possessed a potent in vivo protective effect against juglone-induced oxidative stress in the Caenorhabditis elegans and prolonged its lifespan [8]. Also, Hexahydrofarnesyl acetone had been proven to exhibit a potent antimicrobial and cytotoxic activity [9, 10]. In addition, geranylacetone played an important role in the plant-insects interactions [11], and had a potential effect to inhibit the growth of Gram-positive bacteria [12].

Table 1. Chemical composition of the essential oil of D. Ensifolia

\begin{tabular}{|c|c|c|c|c|}
\hline Compound $^{\mathrm{a}}$ & $\mathbf{R I}^{\mathbf{b}}$ & $\mathbf{R I}^{\mathbf{c}}$ & RI range $^{d}$ & $\%$ \\
\hline Cumene & 921 & $924^{\mathrm{e}}$ & - & 1.3 \\
\hline 1-Octen-3-ol & 978 & $974^{\mathrm{e}}$ & $967-991$ & 1.1 \\
\hline Furfuryl acetate & 990 & $987^{\mathrm{e}}$ & - & 1.4 \\
\hline Linalool & 1098 & $1095^{\mathrm{e}}$ & 1088-1109 & 2.2 \\
\hline (E)-Sabinol & 1140 & $1137^{\mathrm{e}}$ & 1134-1142 & 1.3 \\
\hline$p$-Methoxystyrene & 1155 & $1158^{\mathrm{f}}$ & - & 0.6 \\
\hline (E)-2-Nonenal & 1158 & $1157^{\mathrm{e}}$ & $1154-1173$ & 0.5 \\
\hline$\alpha$-Terpineol & 1190 & $1186^{\mathrm{e}}$ & $1178-1203$ & 1.0 \\
\hline Thymol methyl ether & 1235 & $1232^{\mathrm{e}}$ & - & 0.7 \\
\hline (E)-2-Decenal & 1261 & $1260^{\mathrm{e}}$ & $1255-1276$ & 0.3 \\
\hline Dihydroedulan & 1293 & $1293^{\mathrm{f}}$ & - & 0.6 \\
\hline Theaspirane & 1304 & $1302^{\mathrm{f}}$ & - & 0.3 \\
\hline Eugenol & 1359 & $1356^{\mathrm{e}}$ & $1345-1375$ & 0.8 \\
\hline Silphiperfol-5,7(14)-diene & 1361 & $1358^{\mathrm{e}}$ & - & 0.8 \\
\hline Cyclosativene & 1372 & $1369^{\mathrm{e}}$ & $1360-1380$ & 0.6 \\
\hline$\alpha$-Copaene & 1376 & $1374^{\mathrm{e}}$ & $1363-1391$ & 1.4 \\
\hline 2-epi- $\alpha$-Funebrene & 1381 & $1380^{\mathrm{e}}$ & - & 0.8 \\
\hline Iso-longifolene & 1388 & $1389^{\mathrm{e}}$ & - & 0.6 \\
\hline$\beta$-Elemene & 1392 & $1389^{\mathrm{e}}$ & $1374-1402$ & 1.6 \\
\hline$\alpha$-Gurjunene & 1408 & $1409^{\mathrm{e}}$ & $1394-1421$ & 1.3 \\
\hline Longifolene & 1410 & $1407^{\mathrm{e}}$ & $1387-1434$ & 4.2 \\
\hline$\beta$-Cedrene & 1421 & $1419^{\mathrm{e}}$ & $1415-1434$ & 0.6 \\
\hline$\beta$-Caryophyllene & 1420 & $1417^{\mathrm{e}}$ & $1405-1440$ & 4.0 \\
\hline Aromadendrene & 1437 & $1439^{\mathrm{e}}$ & $1419-1465$ & 0.8 \\
\hline (Z)- $\beta$-Farnesene & 1440 & $1440^{\mathrm{e}}$ & $1438-1460$ & 0.6 \\
\hline$\alpha$-Himachalene & 1447 & $1449^{\mathrm{e}}$ & $1428-1453$ & 0.5 \\
\hline Geranyl acetone & 1452 & $1453^{\mathrm{e}}$ & $1435-1461$ & 6.2 \\
\hline allo-Aromadendrene & 1458 & $1458^{\mathrm{e}}$ & $1443-1477$ & 7.3 \\
\hline Rotundene & 1460 & $1457^{\mathrm{e}}$ & - & 0.8 \\
\hline$\gamma$-Muurolene & 1480 & $1478^{\mathrm{e}}$ & $1461-1487$ & 1.3 \\
\hline$(E)$ - $\beta$-Ionone & 1489 & $1487^{\mathrm{e}}$ & $1470-1498$ & 0.5 \\
\hline
\end{tabular}




\begin{tabular}{|c|c|c|c|c|}
\hline Compound $^{\mathrm{a}}$ & $\mathbf{R I}^{\mathbf{b}}$ & $\mathbf{R \mathbf { I } ^ { \mathbf { c } }}$ & RI range ${ }^{d}$ & $\%$ \\
\hline$\delta$-Selinene & 1494 & $1492^{\mathrm{e}}$ & - & 1.5 \\
\hline$\alpha$-Selinene & 1501 & $1498^{\mathrm{e}}$ & $1477-1510$ & 1.5 \\
\hline$\beta$-Bisabolene & 1507 & $1505^{\mathrm{e}}$ & $1494-1525$ & 1.1 \\
\hline$\delta$-Cadinene & 1523 & $1522^{\mathrm{e}}$ & $1508-1539$ & 1.7 \\
\hline Isoshyobunone & 1533 & $1535^{\mathrm{f}}$ & - & 0.5 \\
\hline (Z)-Sesquisabinene hydrate & 1539 & $1542^{\mathrm{e}}$ & $1524-1562$ & 0.3 \\
\hline Selina-3,7(11)-diene & 1543 & $1545^{\mathrm{e}}$ & $1531-1546$ & 1.8 \\
\hline Elemol & 1549 & $1548^{\mathrm{e}}$ & $1518-1555$ & 0.8 \\
\hline$\beta$-Vetivenene & 1558 & $1554^{\mathrm{e}}$ & - & 1.0 \\
\hline (E)-Nerolidol & 1562 & $1561^{\mathrm{e}}$ & $1539-1570$ & 1.5 \\
\hline Viridiflorol & 1592 & $1592^{\mathrm{e}}$ & $1569-1604$ & 2.2 \\
\hline Cedrol & 1604 & $1600^{\mathrm{e}}$ & $1587-1616$ & 1.9 \\
\hline Widdrol & 1618 & $1617^{\mathrm{f}}$ & - & 1.2 \\
\hline$\alpha$-Cadinol & 1649 & $1652^{\mathrm{e}}$ & $1635-1664$ & 1.6 \\
\hline Methyl jasmonate & 1657 & $1655^{\mathrm{f}}$ & & 0.8 \\
\hline Neointermedeol & 1661 & $1658^{\mathrm{e}}$ & - & 2.6 \\
\hline (E)-2-Tetradecenal & 1674 & $1673^{\mathrm{f}}$ & - & 1.5 \\
\hline 2-Pentadecanone & 1694 & $1697^{\mathrm{e}}$ & $1685-1716$ & 1.0 \\
\hline Pentadecanal & 1713 & $1715^{\mathrm{f}}$ & $1703-1728$ & 1.2 \\
\hline$(2 E, 6 E)$-Farnesal & 1739 & $1740^{\mathrm{e}}$ & - & 0.5 \\
\hline Tetradecanoic acid & 1756 & $1758^{\mathrm{f}}$ & $1749-1782$ & 1.2 \\
\hline Benzyl benzoate & 1763 & $1759^{\mathrm{e}}$ & $1735-1785$ & 1.9 \\
\hline$\beta$-Costol & 1767 & $1765^{\mathrm{e}}$ & - & 1.7 \\
\hline$\alpha$-Costol & 1776 & $1773^{\mathrm{e}}$ & - & 0.9 \\
\hline Neophytadiene & 1838 & $1841^{\mathrm{f}}$ & - & 2.4 \\
\hline Perhydrofarnesyl acetone & 1844 & $1847^{\mathrm{f}}$ & - & 4.4 \\
\hline$(5 E, 9 E)$-Farnesyl acetone & 1915 & $1913^{e}$ & $1918-1921$ & 2.7 \\
\hline Isophytol & 1946 & $1946^{\mathrm{e}}$ & 1939-1951 & 1.1 \\
\hline (E)-15,16-Dinorlabda-8(17),11-dien-13-one & 1994 & $1994^{\mathrm{f}}$ & - & 1.0 \\
\hline Kaurene & 2043 & $2042^{\mathrm{e}}$ & - & 1.8 \\
\hline Oleic Acid & 2141 & $2141^{\mathrm{e}}$ & $2102-2161$ & 2.1 \\
\hline (E)-Phytyl acetate & 2214 & $2218^{e}$ & & 3.8 \\
\hline Total identified & & & & 97.2 \\
\hline
\end{tabular}

${ }^{\mathbf{a}}$ Compounds are listed in order of their elution from a HP-5MS column; ${ }^{\mathbf{b}} \mathbf{R I}$ : Linear retention index relative to $\mathrm{C}_{8}-\mathrm{C}_{30} n$-alkanes on HP-5MS column; ' RI: Relative retention index that refers to Adams (2017) and/or Andriamaharavo (2014). e) [13], f) [14];

d RI range: $90 \%$ confidence retention index range reported by Babushok et al. (2011) [15].

The antibacterial property of the EO was estimated by disc diffusion [16] and micro dilution method [16] and the results are expressed as diameters of inhibition zones (DIZs), minimum inhibitory concentrations (MICs) and minimum bactericidal concentrations (MBCs) in Table 2. The D. ensifolia oil effectively inhibited the growth of all studied bacteria strains with the DIZ values ranged from 10.5 \pm 1.3 to $16.3 \pm 1.7 \mathrm{~mm}$ and the MIC values ranged from 0.16 to $0.31 \mathrm{mg} / \mathrm{mL}$. The MBC/MIC ratio is no more than 2, which suggested that the D. ensifolia essential oil presented a good bactericidal effect for the tested bacterial strains. The good antibacterial activity of the $D$. ensifolia essential oil observed may be associated with the presence of geranylacetone, hexahydrofarnesyl acetone, longifolene and $\beta$ caryophyllene, which were reported to have antibacterial effects against many bacterial strains $[9,12,17$, 18]. However, synergistic effect between constituents should not be neglected, since it may cause a much more noticeable effect than single component [19-20]. 
Table 2. Antibacterial activity of D. ensifolia essential oil

\begin{tabular}{|c|c|c|c|c|c|c|}
\hline \multirow[b]{2}{*}{ Test strains } & \multicolumn{2}{|c|}{$\begin{array}{c}\text { a'Diameter of the inhibition } \\
\text { zones }(\mathrm{mm})\end{array}$} & \multicolumn{2}{|c|}{ MIC $(\mathrm{mg} / \mathrm{mL})$} & \multicolumn{2}{|c|}{$\mathrm{MBC}(\mathrm{mg} / \mathrm{mL})$} \\
\hline & $\begin{array}{c}\text { EO } \\
(10 \mu \mathrm{g} / \mathrm{disk})\end{array}$ & $\begin{array}{c}{ }^{\mathrm{a}} \mathrm{Ch} \\
(0.1 \mu \mathrm{g} / \mathrm{disk})\end{array}$ & EO & Ch & EO & Ch \\
\hline \multicolumn{7}{|l|}{ Gram positive } \\
\hline Staphylococcus aureus ATCC 6538 & $15.5 \pm 1.4$ & $32.1 \pm 1.2$ & 0.16 & 0.004 & 0.16 & 0.16 \\
\hline Bacillus subtilis ATCC 6633 & $10.5 \pm 1.3$ & $36.5 \pm 2.5$ & 0.31 & 0.002 & 0.63 & 0.02 \\
\hline Gram negative & & & & & & \\
\hline Escherichia coli ATCC 25922 & $16.3 \pm 1.7$ & $35.8 \pm 2.3$ & 0.16 & 0.002 & 0.31 & 0.06 \\
\hline Pseudomonas aeruginosa ATCC 27853 & $11.1 \pm 0.9$ & $13.8 \pm 1.4$ & 0.31 & 0.125 & 0.63 & 2.50 \\
\hline
\end{tabular}

The antioxidant capacity of the EO was examined using various methods, namely, 2,2-diphenyl1-picrylhydrazyl (DPPH) radical scavenging, 2,20-azinobis-3-ethylbenzothiazoline-6-sulphonate (ABTS) cation radical scavenging, and ferric reducing antioxidant potential (FRAP) assays [16, 20]. Trolox and BHT (butylated hydroxytoluene) were used as the positive controls. The results of DPPH assay demonstrated that the $D$. ensifolia essential oil showed a weak radical scavenging activity with $\mathrm{IC}_{50}$ of $1.37 \mathrm{mg} / \mathrm{mL}$, which was much higher than the $\mathrm{IC}_{50}$ values of reference standards, $\mathrm{BHT}$ ( $\mathrm{IC}_{50}$ of $0.005 \mathrm{mg} / \mathrm{mL}$ ) and Trolox $\left(\mathrm{IC}_{50}\right.$ of $0.036 \mathrm{mg} / \mathrm{mL}$ ). Nevertheless, a moderate antioxidant activity was observed in the ABTS assay $\left(\mathrm{IC}_{50}\right.$ value of $0.13 \mathrm{mg} / \mathrm{mL}$ ). In FRAP assay, the ferric ion reducing capacity was expressed in TEAC (Trolox equivalent antioxidant concentration) units ( $\mu$ mol Trolox $\times \mathrm{g}^{-1} \mathrm{EO}$ ). The result revealed a moderate reducing activity of the EO $\left(\right.$ TEAC $=134.70 \mu \mathrm{mol}$ Trolox $\left.\times \mathrm{g}^{-1}\right)$. The most abundant compounds in the $D$. ensifolia essential oil, viz., allo-aromadendrene, geranylacetone, and $\beta$-caryophyllene have been reported to exhibit antioxidant activity $[8,11,18]$.

Table 3. In vitro antioxidant activity (DPPH, ABTS and FRAP) of D. ensifolia essential oil

\begin{tabular}{cccc}
\hline Sample & DPPH IC $\mathbf{5 0}(\mathbf{m g} / \mathbf{m L})$ & ABTS IC $_{\mathbf{5 0}}(\mathbf{m g} / \mathbf{m L})$ & FRAP $\left(\boldsymbol{\mu m o l ~ T r o l o x} \times \mathbf{g}^{\mathbf{- 1}}\right)$ \\
\hline EO & $1.37 \pm 0.12$ & $0.13 \pm 0.02$ & $134.70 \pm 5.56$ \\
BHT & $0.005 \pm 0.001$ & $0.003 \pm 0.001$ & \\
Trolox & $0.036 \pm 0.005$ & $0.038 \pm 0.004$ & \\
\hline
\end{tabular}

The cytotoxicity of the EO was evaluated against HepG2 (liver hepatocellular cells) and MCF-7 (human breast adenocarcinoma cell line) cell lines using the MTT [3-(4,5-dimethylthiazol-2-yl)-2,5diphenyltetrazolium bromide] assay [16]. Doxorubicin was used as the positive control. The result is given in Table 4. The essential oil of $D$. ensifolia exerted a dose dependent cytotoxic effect on HepG2 and MCF-7 tumor cell lines with $\mathrm{IC}_{50}$ values of $(61.35 \pm 14.57) \mu \mathrm{g} / \mathrm{mL}$ and $(56.53 \pm 11.54) \mu \mathrm{g} / \mathrm{mL}$, respectively. The interesting cytotoxic activity toward cell lines could be due to the main compounds in the essential oil. In addition to allo-aromadendrene and hexahydrofarnesyl acetone, literature reported that $\beta$-caryophyllene also showed potential ability to inhibit tumour motility, cell invasion and tumour aggregation [18].

Table 4. Cytotoxicity of D. ensifolia essential oil against HepG2 and MCF-7 cells

\begin{tabular}{cccccc}
\hline & \multicolumn{2}{c}{ HepG2 } & $\mathbf{I C}_{\mathbf{5 0}}(\boldsymbol{\mu g} / \mathbf{m L})$ & & \multicolumn{2}{c}{ MCF-7 } & $\mathbf{I C}_{\mathbf{5 0}}(\boldsymbol{\mu g} \mathbf{g} \mathbf{m L})$ \\
\cline { 2 - 3 } \cline { 5 - 6 } & EO & Doxorubicin & & EO & Doxorubicin \\
\hline $24 \mathrm{~h}$ & $110.33 \pm 13.64$ & $1.43 \pm 0.24$ & & $93.68 \pm 6.32$ & $0.61 \pm 0.23$ \\
$48 \mathrm{~h}$ & $92.13 \pm 6.65$ & $0.48 \pm 0.01$ & & $78.63 \pm 6.68$ & $0.18 \pm 0.07$ \\
$72 \mathrm{~h}$ & $61.35 \pm 14.57$ & $0.27 \pm 0.04$ & & $56.53 \pm 11.54$ & $0.07 \pm 0.02$ \\
\hline
\end{tabular}




\section{Supporting Information}

Supporting Information accompanies this paper on http://www.acgpubs.org/journal/recordsof-natural-products

\section{ORCID}

Zi-Qian He: 0000-0002-9602-5557

Xue-Yuan Shen: 0000-0001-8373-8155

Ze-Yu Cheng: 0000-0002-3302-3378

Ruo-Lan Wang: 0000-0002-2870-7652

Peng-Xiang Lai: 0000-0002-5380-4382

Xiang Xing: 0000-0003-0951-5708

\section{References}

[1] Chinese Academy of Sciences. (1980) Flora Reipublicae Popularis Sinicae. Science Press, Beijing, 14, pp.35.

[2] B. Q. Tang, C. W. Li, J. B. Sun, Y. Chang, J. Y. W. Chan, S. M. Y. Lee and B. Zeng (2017). A new cycloartane-type triterpenoid from the roots of Dianella ensifolia (L.) DC, Nat. Prod. Res. 31, 966-971.

[3] B. Q. Tang, S. S. Huang, Y. E. Liang, J. B. Sun, Y. Ma, B. Zeng, S. M. Y. Lee and J. L. Lu (2017). Two new flavans from the roots of Dianella ensifolia (L.) DC, Nat. Prod. Res. 31, 1561-1565.

[4] R. Randrianasolo, A. Raharinirina, H. L. Rasoanaivo, H. C. Krebs, A. Raharisolololao, A. A. Razakarivony and M. F. Rakotondramanga (2015). A new Dihydronaphtaquinone from Dianella ensifolia L. Redout, J. Pharmacogn. Phytochem. 3, 140-144.

[5] T. Mammone, N. Muizzuddin, L. Declercq, D. Clio, H. Corstjens, I. Sente, K. Van Rillaer, M. Matsui, Y. Niki, M. Ichihashi, P. U. Giacomoni and D. Yarosh (2010). Modification of skin discoloration by a topical treatment containing an extract of Dianella ensifolia: a potent antioxidant, J. Cosmet. Dermatol. 9, 89-95.

[6] A. Nesterov, J. Zhao, D. Minter, C. Hertel, W. Ma, P. Abeysinghe, M. Hong and Q. Jia (2008). 1-(2, 4Dihydroxyphenyl)-3-(2, 4-dimethoxy-3-methylphenyl) propane, a novel tyrosinase inhibitor with strong depigmenting effects, Chem. Pharm. Bull. 56, 1292-1296.

[7] S. S. Sawant, D. T. A. Youssef, P. W. Sylvester, V. Wali and K. A. EI Sayed (2007). Antiproliferative sesquiterpenes from the Red Sea soft coral Sarcophyton glaucum, Nat. Prod. Commun. 2, 117-119.

[8] C. W. Yu, W. H. Li, F. L. Hsu, P. L. Yen, S. T. Chang and V. H. C. Liao (2014). Essential oil alloaromadendrene from mixed-type Cinnamomum osmophloeum leaves prolongs the lifespan in Caenorhabditis elegans, J. Agr. Food Chem. 62, 6159-6165.

[9] N. Filipowicz, M. Kamiński, J. Kurlenda, M. Asztemborska and J. R. Ochocka (2003). Antibacterial and antifungal activity of juniper berry oil and its selected components, Phytother. Res. 17, 227-231.

[10] S. M. Razavi and S. Nejad-Ebrahimi (2010). Phytochemical analysis and allelopathic activity of essential oils of Ecballium elaterium A. Richard growing in Iran, Nat. Prod. Res. 24, 1704-1709.

[11] A. Stobiecka (2015). Comparative study on the free radical scavenging mechanism exerted by geraniol and geranylacetone using the combined experimental and theoretical approach, Flavour Frag. J. 30, 399409.

[12] R. Bonikowski, P. Świtakowska and J. Kula (2015). Synthesis, odour evaluation and antimicrobial activity of some geranyl acetone and nerolidol analogues, Flavour Frag. J. 30, 238-244.

[13] R. P. Adams (2017). Identification of essential oil components by gas chromatography/mass spectrometry. 5th Ed. Texensis Publishing Gruver, TX USA.

[14] N. R. Andriamaharavo (2014). Retention Data NIST Mass Spectrometry Data Center, NIST Mass Spectrometry Data Center.

[15] V.I. Babushok, P.J. Linstrom and I.G. Zenkevich (2011). Retention indices for frequently reported compounds of plant essential oils. J. Phys. Chem. Ref. Data. 40(4), 1-47.

[16] X. D. Su, Yang Gao, Y. X. Xiang, P. X. Lai and X. Xing (2019). Chemical composition and biological activities of the essential oil from Aristolochia fordiana Hemsl, Rec. Nat. Prod. 13, 346-354. 
[17] A. Y. Gordien, A. I. Gray, S. G. Franzblau and V. Seidel (2009). Antimycobacterial terpenoids from Juniperus communis L.(Cuppressaceae), J. Ethnopharmacol. 126, 500-505.

[18] S. Dahham, Y. Tabana, M. Iqbal, M. Ahamed, M. Ezzat, A. Majid and A. Majid (2015). The anticancer, antioxidant and antimicrobial properties of the sesquiterpene $\beta$-caryophyllene from the essential oil of Aquilaria crassna, Molecules 20, 11808-11829.

[19] M. A. Oukerrou, M. Tilaoui, H. A. Mouse, I. Leouifoudi, A. Jaafari and A. Zyad (2017). Chemical composition and cytotoxic and antibacterial activities of the essential oil of Aloysia citriodora palau grown in Morocco, Adv. Pharmacol. Sci. 2017, 7801924.

[20] L. J. Mao, X. Y. Xie, Y. Gao and P. X. Lai (2019). Chemical composition, antibacterial and antioxidant activities of essential oil from Leonurus pseudomacranthus Kitag, Rec. Nat. Prod. 13, 91-95.

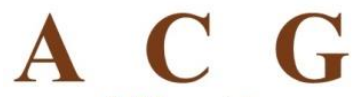

publications

(C) 2019 ACG Publications 